\title{
Severe mental illness and chronic kidney disease: a cross-sectional study in the United Kingdom
}

This article was published in the following Dove Press journal:

Clinical Epidemiology

\author{
Masao Iwagami' \\ Kathryn E Mansfield' \\ Joseph F Hayes ${ }^{2}$ \\ Kate Walters ${ }^{3}$ \\ David PJ Osborn 2,4 \\ Liam Smeeth' \\ Dorothea Nitsch' \\ Laurie A Tomlinson' \\ 'Department of Non-Communicable \\ Disease Epidemiology, London School \\ of Hygiene and Tropical Medicine, \\ London, UK; ${ }^{2}$ Division of Psychiatry, \\ University College London, London, \\ UK; ${ }^{3}$ Department of Primary Care and \\ Population Health, University College \\ London, London, UK; ${ }^{4}$ Camden and \\ Islington NHS Foundation Trust, \\ London, UK
}

Correspondence: Masao Iwagami Department of Non-Communicable Disease Epidemiology, London School of Hygiene and Tropical Medicine, Keppel Street, London WCIE 7HT, UK Email masao.iwagami@Ishtm.ac.uk
Objective: We investigated the burden of chronic kidney disease (CKD) among patients with severe mental illness (SMI).

Methods: We identified patients with SMI among all those aged 25-74 registered in the UK Clinical Practice Research Datalink as on March 31, 2014. We compared the prevalence of CKD (two measurements of estimated glomerular filtration rate $<60 \mathrm{~mL} / \mathrm{min} / 1.73 \mathrm{~m}^{2}$ for $\geq 3$ months) and renal replacement therapy between patients with and without SMI. For patients with and without a history of lithium prescription separately, we used logistic regression to examine the association between SMI and CKD, adjusting for demographics, lifestyle characteristics, and known CKD risk factors.

Results: The CKD prevalence was $14.6 \%$ among patients with SMI and a history of lithium prescription ( $\mathrm{n}=4,295), 3.3 \%$ among patients with SMI and no history of lithium prescription ( $\mathrm{n}=24,101)$, and $2.1 \%$ among patients without SMI $(\mathrm{n}=2,387,988 ; P<0.001)$. The prevalence of renal replacement therapy was $0.23 \%, 0.15 \%$, and $0.11 \%$, respectively $(P=0.012)$. Compared to patients without SMI, the fully adjusted odds ratio for CKD was 6.49 (95\% CI 5.84-7.21) for patients with SMI and a history of lithium prescription and 1.45 (95\% CI 1.34-1.58) for patients with SMI and no history of lithium prescription. The higher prevalence of CKD in patients with SMI may, in part, be explained by more frequent blood testing as compared to the general population. Conclusion: CKD is identified more commonly among patients with SMI than in the general population.

Keywords: severe mental illness, schizophrenia, bipolar disorder, chronic kidney disease, lithium

\section{Introduction}

People with severe mental illness (SMI), including schizophrenia, bipolar disorder, and other nonorganic psychotic illnesses, are known to have shorter life expectancy, by $\sim 10-20$ years, than the general population. ${ }^{1-6}$ Their premature death is attributed not only to suicide and accident but also to physical illnesses - especially cardiovascular diseases such as coronary heart disease and stroke. ${ }^{5-9}$ The high rate of cardiovascular deaths in patients with SMI has been attributed to the high prevalence of lifestylerelated conditions (eg, smoking and obesity), ${ }^{10-13}$ suboptimal screening/assessment of cardiovascular risks, ${ }^{14,15}$ and suboptimal management of underlying diseases (eg, hypertension, diabetes, dyslipidemia). ${ }^{16-18}$

Chronic kidney disease (CKD) - the continuous presence of kidney damage and/or decreased level of kidney function ${ }^{19}$ - is independently associated with increased risk of death, cardiovascular events, and all-cause hospitalization. ${ }^{20} \mathrm{CKD}$ 
can progress to end-stage renal disease (ESRD) requiring renal replacement therapy (RRT), a substantial burden for both - quality of life of patients and health budgets. The UK National Institute for Health and Care Excellence guidance for CKD recommends early identification and appropriate management of CKD. ${ }^{21}$

People with SMI have an increased prevalence of several risk factors for CKD, including smoking and diabetes. ${ }^{13,16,22}$ Moreover, patients with SMI may be treated with lithium, which is associated with the development of CKD. ${ }^{23,24}$ However, there has been limited research investigating the prevalence of, and risk factors for, CKD in people with SMI. ${ }^{13,25,26}$ In the UK, primary care plays an important role in the management of physical health conditions in people with SMI. As part of the Quality and Outcomes Framework (QOF), there have been recent financial incentives for general practitioners (GPs) to undertake physical health checks in people with SMI. ${ }^{27}$ However, it is unclear whether CKD in patients with SMI is appropriately recognized and managed by GPs.

Therefore, we used a UK primary care database to: 1) compare the prevalence of CKD and RRT among patients with SMI (with and without a history of lithium use) and those without SMI; 2) investigate whether there is an independent association between SMI and CKD after adjusting for known CKD risk factors; and 3) compare the recognition and management of CKD between CKD patients with and without SMI.

\section{Materials and methods Data source}

The Clinical Practice Research Datalink (CPRD) is an observational data and interventional research service provided by the National Health Service. Around 98\% of the UK population are registered with a primary care practice. Currently, $>650$ general practices contribute data conforming to quality control standards to the CPRD, including 7\% of the UK population. The included practices are representative of the UK general population in terms of age and sex. ${ }^{28}$ The database includes the following data: patient demographics, diagnoses based on Read codes (a hierarchical coding system), prescriptions based on British National Formulary codes, laboratory test results, and referrals made by GPs. Ethical approval for this study was obtained from the Independent Scientific Advisory Committee of CPRD (protocol no. 16_055). Informed consent was waived because data are anonymized for research purposes.

\section{Study population}

Our study population included all people, aged 25-74 years, registered in the CPRD for at least 1 year as on March 31, 2014 (ie, the end of a financial or QOF year). We selected the 25-74 age range because our preliminary analysis suggested that CKD and/or SMI were rare in people outside these parameters, thereby limiting the power for comparative prevalence analyses. To ensure that we had reliable measures of morbidity (to allow time for the recording of past medical history in newly registered patients), we required that all participants had at least 1 year of continuous registration in the CPRD.

\section{Disease definition}

We identified SMI using Read morbidity codes that have been validated in UK primary care data, with high sensitivity (91\%), specificity (99.9\%), and positive predictive value $(91 \%) .{ }^{29}$ Patients with SMI were further classified as those with and without any record of lithium prescription in the period between the CPRD registration and March 31, 2014. We excluded patients who had been prescribed lithium without recorded SMI from the general population comparison group because the indication for treatment was not known.

Our definition of CKD was based on two measurements of estimated glomerular filtration rate $<60 \mathrm{~mL} / \mathrm{min} / 1.73 \mathrm{~m}^{2}$ separated by 3 months or longer, ${ }^{19}$ calculated from serum creatinine records in the CPRD in the past 5 years, using the Chronic Kidney Disease Epidemiology Collaboration equation. ${ }^{30}$ RRT was characterized on the basis of diagnosis codes suggesting hemodialysis, peritoneal dialysis, or kidney transplantation. Previous research has shown that the prevalence of estimated glomerular filtration rate $<60 \mathrm{~mL} / \mathrm{min} / 1.73 \mathrm{~m}^{2}$ and RRT in the CPRD is similar to that estimated in a nationally representative population survey (Health Survey for England) and disease registry (UK Renal Registry), suggesting that most cases of CKD and RRT are captured in the CPRD. ${ }^{31}$

\section{Covariate definition}

We defined baseline characteristics of patients with and without SMI: age, sex, ethnicity, socioeconomic status (SES), country of the UK (ie, England, Northern Ireland, Scotland, and Wales) body mass index (BMI), smoking status, chronic diseases that are associated with $\mathrm{CKD}^{21}$ - diabetes, hypertension, cardiovascular disease (ie, myocardial infarction, chronic heart failure, peripheral arterial disease, and stroke), urological disease (ie, vesicoureteral reflux, renal tract stone, and prostatic hypertrophy), systematic lupus erythematosus, and polycystic kidney disease - based on relevant diagnosis 
codes. Based on previous studies using UK primary care data, ${ }^{32,33}$ we grouped patients with no record of ethnicity into those of white ethnicity. SES was assigned at the general practice level using quintiles of the Index of Multiple Deprivation in each country of the UK. ${ }^{34}$ BMI and smoking status were based on the most recent records prior to the date of study inclusion (ie, March 31, 2014). For chronic diseases, if a diagnosis code was recorded for a patient during the period between CPRD registration and the study date, then that disease was regarded as being present.

\section{Data analysis}

We compared baseline characteristics and overall prevalence of CKD (including patients on RRT) among the three groups: patients with SMI with and without a history of lithium prescription as well as those without SMI, using chi-squared tests. We stratified CKD prevalence by age (using 10-year age bands) and sex. We estimated the prevalence of RRT among the three groups and compared it overall by chi-squared tests. Moreover, we compared the distribution of the most recent category of RRT modality (hemodialysis, peritoneal dialysis, or kidney transplantation) between patients with and without SMI using chi-squared tests.

Next, we carried out logistic regression analyses to examine the association between SMI (with and without a history of lithium use) and CKD. To understand which factors are more likely to explain the association between SMI and CKD, we adjusted step-by-step for age and sex, ethnicity, SES, BMI, smoking status, and chronic diseases associated with decline in kidney function (ie, diabetes, hypertension, cardiovascular disease, urological disease, systemic lupus erythematosus, and polycystic kidney disease). ${ }^{21}$ Patients with missing data of $\mathrm{BMI}$ and smoking status were excluded from the analysis. We took into account variations in coding and testing practices by different general practices through clustering by general practice in the logistic regression models.

Finally, we restricted analysis to patients with CKD who were not on RRT and compared the recognition and management of CKD between patients with and without SMI. We used the QOF CKD indicators (QOF version 2013/14) as markers of the recognition and management of CKD in the UK primary care. ${ }^{27}$ We determined the proportion of CKD patients with and without SMI who had: 1) a record of a diagnostic code for $\mathrm{CKD}$, using CKD codes listed in the QOF version $2013 / 14 ;{ }^{35} 2$ ) the most recent blood pressure measure $\leq 140 / 90 \mathrm{mmHg}$ (recorded in the year before the date of the study); 3) urine test for proteinuria/albuminuria (including dipstick test) in the year before the date of the study; and 4) for those with hypertension and proteinuria (recorded any time between CPRD registration and study date), at least one prescription for a renin-angiotensin system antagonist in the past 3 months. In addition, because the National Institute for Health and Care Excellence guidance for cardiovascular disease recommends statins as primary prevention for all CKD patients regardless of serum cholesterol levels, ${ }^{36}$ we compared the proportion of CKD patients with and without SMI who had at least one prescription for a statin in the past 3 months.

All statistical analyses were conducted using Stata 14 (Stata Corp, College Junction, TX, USA).

\section{Results}

Of the 2,418,730 people aged 25-74 years who were registered in the CPRD for $>1$ year on March 31, 2014, we identified 28,396 (1.17\%) patients with SMI, including 4,295 patients with a history of lithium prescription in the CPRD and 24,101 without (Figure 1). In patients with SMI (both with and without

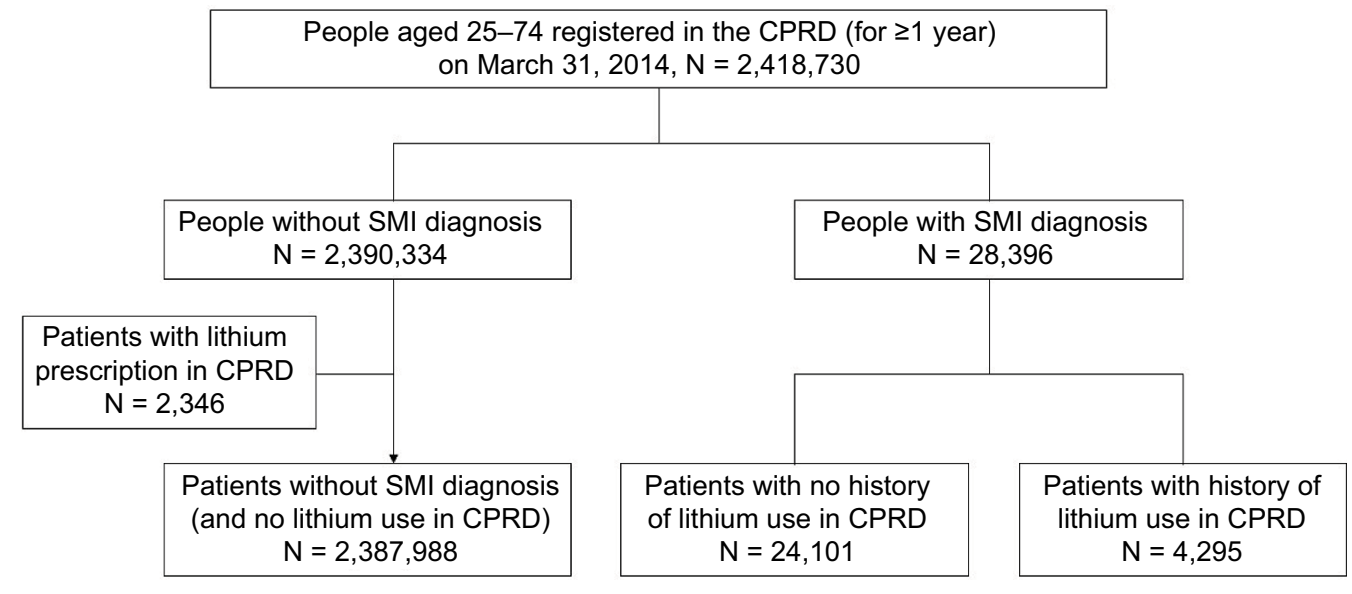

Figure I Flow chart showing identification of study participants. Abbreviations: CPRD, Clinical Practice Research Datalink; SMI, severe mental illness. 
a previous lithium prescription) compared to those without, the SES was generally lower and the proportions of black ethnicity, obesity (BMI $\geq 30 \mathrm{~kg} / \mathrm{m}^{2}$ ), and current smokers were higher. Diabetes, hypertension, and cardiovascular diseases were more common in patients with SMI, whereas the prevalence of other diseases (urological disease, systematic lupus erythematosus, and polycystic kidney disease) was similar between patients with and without SMI (Table 1).

Overall, the prevalence of CKD was $14.64 \%(629 / 4,295)$ in patients with SMI and history of lithium prescription, $3.34 \%(805 / 24,101)$ in patients with SMI and no history of lithium prescription, and $2.09 \%(49,870 / 2,387,988)$ in patients without SMI $(P<0.001)$. The absolute difference in CKD prevalence among the three groups increased with age in both men and women (Figure 2). Moreover, there was evidence $(P=0.012)$ that the overall prevalence of RRT was higher in patients with SMI $(0.23 \%[10 / 4,295]$ and $0.15 \%$ $[36 / 24,101]$ in those with and without a history of lithium prescription, respectively) than those without SMI $(0.11 \%$ $[2,645 / 2,387,988])$. There was strong evidence that the distribution of RRT modalities was different between patients with and without SMI $(P=0.001)$. In patients with SMI, $50 \%(23 / 46)$ were receiving hemodialysis and $2 \%(1 / 46)$ peritoneal dialysis, and 48\% (22/46) underwent kidney transplantation. However, in patients without SMI, the corresponding figures were $26 \%(700 / 2,645), 7 \%(194 / 2,645)$, and $66 \%(1,751 / 2,645)$.

The age-sex-adjusted odds ratio (OR) for CKD was 7.13 (95\% confidence interval [CI] 6.47-7.85) for patients with SMI and history of lithium use, and 1.69 (1.56-1.83) for those with SMI and no history of lithium use, compared to those without SMI. After adjusting for SES, BMI, smoking, and chronic diseases associated with CKD, the adjusted ORs decreased to $6.49(5.84-7.21)$ and $1.45(1.34-1.58)$ but were still significant in patients with SMI with and without previous lithium prescription, respectively (Table 2).

Among patients with biochemically defined CKD (not on RRT), a higher proportion of people with SMI had a recorded diagnosis of CKD (SMI 70.3\%, without SMI 65.5\%) and a recorded blood pressure $\leq 140 / 90 \mathrm{mmHg}$ (SMI 80.1\%, without SMI 75.6\%; Table 3). However, a lower proportion of patients with SMI had recently been prescribed statins (SMI 50.5\%, without SMI 59.0\%).

\section{Discussion \\ Main findings}

We found that, in UK primary care, patients with SMI had a greater prevalence of CKD compared to the general population. This was pronounced (6.5-fold increase) in patients with a history of lithium prescription, but there was a 1.5-fold increase in odds of CKD even among patients never known to be prescribed lithium and after adjustment for differences in known risk factors for CKD. In addition, patients with SMI had an increased prevalence of RRT and were more likely to be receiving hemodialysis as their modality than patients without SMI on RRT.

\section{Strengths and limitations}

There are several strengths of our study. There is likely to be a good recording of the diagnoses examined because GPs manage patients with SMI for their physical and mental health - with or without the support of psychiatrists in secondary care. In addition, the QOF - the reward and incentive program for GPs - included both SMI and CKD during this time period. ${ }^{27}$ Moreover, the diagnosis of SMI has been validated at the individual level, ${ }^{29}$ and the prevalence estimates of CKD and RRT have been validated at the population level. ${ }^{31}$

However, we need to acknowledge several limitations. First, this is a cross-sectional study, in which temporal relationships cannot always be clarified. The majority of patients with SMI have developed symptoms by early adulthood, ${ }^{37}$ whereas the prevalence of CKD starts to increase after age $55 .{ }^{31}$ Therefore, it is unlikely that CKD precedes the onset of SMI. However, the interpretation of potential risk factors in the association between SMI and CKD requires caution. For example, cessation of smoking and lithium may be as a result of the development of CKD. Therefore, we did not differentiate previous and current users in the regression models. Second, a greater prevalence of CKD among patients with SMI may, in part, be influenced by surveillance or ascertainment bias. Patients with SMI take medications, such as lithium and other psychotropic drugs, which need regular monitoring. In addition, in 2013-2014, GPs were incentivized to monitor blood glucose and cholesterol levels for people with SMI; therefore, many patients would have had concurrent testing of renal function. ${ }^{38}$ In contrast, in the general population, creatinine testing in primary care is not universal: in 2013-2014, this was recommended and incentivized only for people with known CKD risk factors. ${ }^{21,27}$ However, we have previously demonstrated that the prevalence of CKD identified in the CPRD was very similar to that seen in the Health Survey for England, a nationally representative survey of the general population. ${ }^{31}$ This suggests that most patients with CKD are captured by the current testing strategy in primary care, and the proportion of patients with unmeasured CKD in CPRD is small. Therefore, underascertainment of CKD in people 
Table I Characteristics of patients by SMI diagnosis and history of lithium use

\begin{tabular}{|c|c|c|c|}
\hline \multirow[t]{3}{*}{ Characteristic } & \multirow{2}{*}{$\begin{array}{l}\text { Patients without } \\
\text { SMI diagnosis } \\
N=2,387,988\end{array}$} & \multicolumn{2}{|c|}{ Patients with SMI diagnosis $(\mathrm{N}=28,396)$} \\
\hline & & $\begin{array}{l}\text { Patients with no history of } \\
\text { lithium use } N=24,101\end{array}$ & $\begin{array}{l}\text { Patients with history of } \\
\text { lithium use } N=4,295\end{array}$ \\
\hline & n (\%) & n (\%) & n (\%) \\
\hline \multicolumn{4}{|l|}{ Type of SMI diagnosis } \\
\hline Schizophrenia & - & $9,134(37.9)$ & $448(10.4)$ \\
\hline Bipolar disorder & - & $5,881(24.4)$ & $3,374(78.6)$ \\
\hline Other nonorganic psychosis & - & $9,086(37.7)$ & $473(11.0)$ \\
\hline \multicolumn{4}{|l|}{ Age category (years) } \\
\hline $25-34$ & $468,602(19.6)$ & $3,654(15.2)$ & $242(5.6)$ \\
\hline $35-44$ & $503,405(21.1)$ & $5,475(22.7)$ & $688(16.0)$ \\
\hline $45-54$ & $571,325(23.9)$ & $6,361(26.4)$ & $\mathrm{I}, 187(27.6)$ \\
\hline $55-64$ & $456,457(19.1)$ & $4,844(20.1)$ & $1,209(28.2)$ \\
\hline $65-74$ & $388,199(16.3)$ & $3,767(15.6)$ & $969(22.6)$ \\
\hline Sex (male) & $1,193,218(50.0)$ & I3, I83 (54.7) & $I, 761(4 I .0)$ \\
\hline \multicolumn{4}{|l|}{ Ethnicity } \\
\hline White/unrecorded* & $2,232,403(93.5)$ & $22,05 ।(91.5)$ & $4,184(97.4)$ \\
\hline Black & $42,339(1.8)$ & $836(3.5)$ & $35(0.8)$ \\
\hline South Asian & $72,190(3.0)$ & $734(3.1)$ & $42(1.0)$ \\
\hline Other & $41,056(1.7)$ & $480(2.0)$ & $34(0.8)$ \\
\hline \multicolumn{4}{|l|}{ Country } \\
\hline England & $1,739,874(72.9)$ & I7,507 (72.6) & $2,815(65.5)$ \\
\hline North Ireland & $92,714(3.9)$ & $931(3.9)$ & $234(5.5)$ \\
\hline Scotland & $307,936(12.9)$ & $3,084(12.8)$ & $765(17.8)$ \\
\hline Wales & $247,464(10.4)$ & $2,579(10.7)$ & $481(11.2)$ \\
\hline \multicolumn{4}{|l|}{ Socioeconomic status } \\
\hline I (least deprived) & $468,867(19.6)$ & $3,463(14.4)$ & $856(19.9)$ \\
\hline 2 & $496,229(20.8)$ & $4,295(17.8)$ & $864(20.1)$ \\
\hline 3 & $483,617(20.3)$ & $4,706(19.5)$ & $833(19.4)$ \\
\hline 4 & $508,424(21.3)$ & $6,084(25.2)$ & $948(22.1)$ \\
\hline 5 (most deprived) & $430,851(18.0)$ & $5,553(23.0)$ & $794(18.5)$ \\
\hline \multicolumn{4}{|l|}{ Body mass index $\left(\mathrm{kg} / \mathrm{m}^{2}\right)$} \\
\hline$<18.5$ & $37,526(1.6)$ & $497(2.1)$ & $56(1.3)$ \\
\hline 18.5 to $<25$ & $772,208(32.3)$ & $6,868(28.5)$ & $988(23.0)$ \\
\hline$\geq 25$ to $<30$ & $716,303(30.0)$ & $7,480(31.0)$ & $\mathrm{I}, 482(34.5)$ \\
\hline$\geq 30$ & $530,065(22.2)$ & $8,281(34.4)$ & $\mathrm{I}, 724(40.1)$ \\
\hline Missing data & $331,886(13.9)$ & $975(4.1)$ & $45(I . I)$ \\
\hline \multicolumn{4}{|l|}{ Smoking status } \\
\hline Nonsmoker & $962,06 \mathrm{I}(40.3)$ & $5,578(23.1)$ & $1,123(26.2)$ \\
\hline Current smoker & $516,109(21.6)$ & $10,677(44.3)$ & I,485 (34.6) \\
\hline Ex-smoker & $86 I, 4 \mid 4(36.1)$ & $7,773(32.3)$ & $1,685(39.2)$ \\
\hline Missing data & $48,404(2.0)$ & $73(0.3)$ & $<5(<0.1)$ \\
\hline \multicolumn{4}{|c|}{ Chronic diseases associated with CKD } \\
\hline Diabetes mellitus & $166,917(7.0)$ & $3,42 \mid(\mid 4.2)$ & $718(16.7)$ \\
\hline Hypertension & $682,868(28.6)$ & $8,826(36.6)$ & $2,138(49.8)$ \\
\hline \multicolumn{4}{|l|}{ Cardiovascular disease } \\
\hline Myocardial infarction & $31,659(1.3)$ & $390(1.6)$ & $81(1.9)$ \\
\hline Chronic heart failure & $10,997(0.5)$ & $192(0.8)$ & $38(0.9)$ \\
\hline Peripheral arterial disease & $15,383(0.6)$ & $232(1.0)$ & $47(1.1)$ \\
\hline Stroke & $24,097(1.0)$ & $419(1.7)$ & $96(2.2)$ \\
\hline \multicolumn{4}{|l|}{ Urological disease } \\
\hline Vesicoureteral reflux & $1,676(0.1)$ & $16(0.1)$ & $5(0.1)$ \\
\hline Renal tract stone & $30,665(1.3)$ & $266(1.1)$ & $41(1.0)$ \\
\hline Prostatic hypertrophy & $40,855(1.7)$ & $379(1.6)$ & $105(2.4)$ \\
\hline \multicolumn{4}{|l|}{ Other } \\
\hline Systemic lupus erythematosus & $3,423(0.1)$ & $47(0.2)$ & $5(0.1)$ \\
\hline Polycystic kidney disease & $\mathrm{I}, 980(0.1)$ & $22(0.1)$ & $<5(<0.1)$ \\
\hline
\end{tabular}

Note: *Number of patients without recorded ethnicity was I, I4I,478 (47.8\%), 9,687 (40.2\%), and 2,045 (47.6\%), respectively.

Abbreviations: CKD, chronic kidney disease; SMI, severe mental illness. 


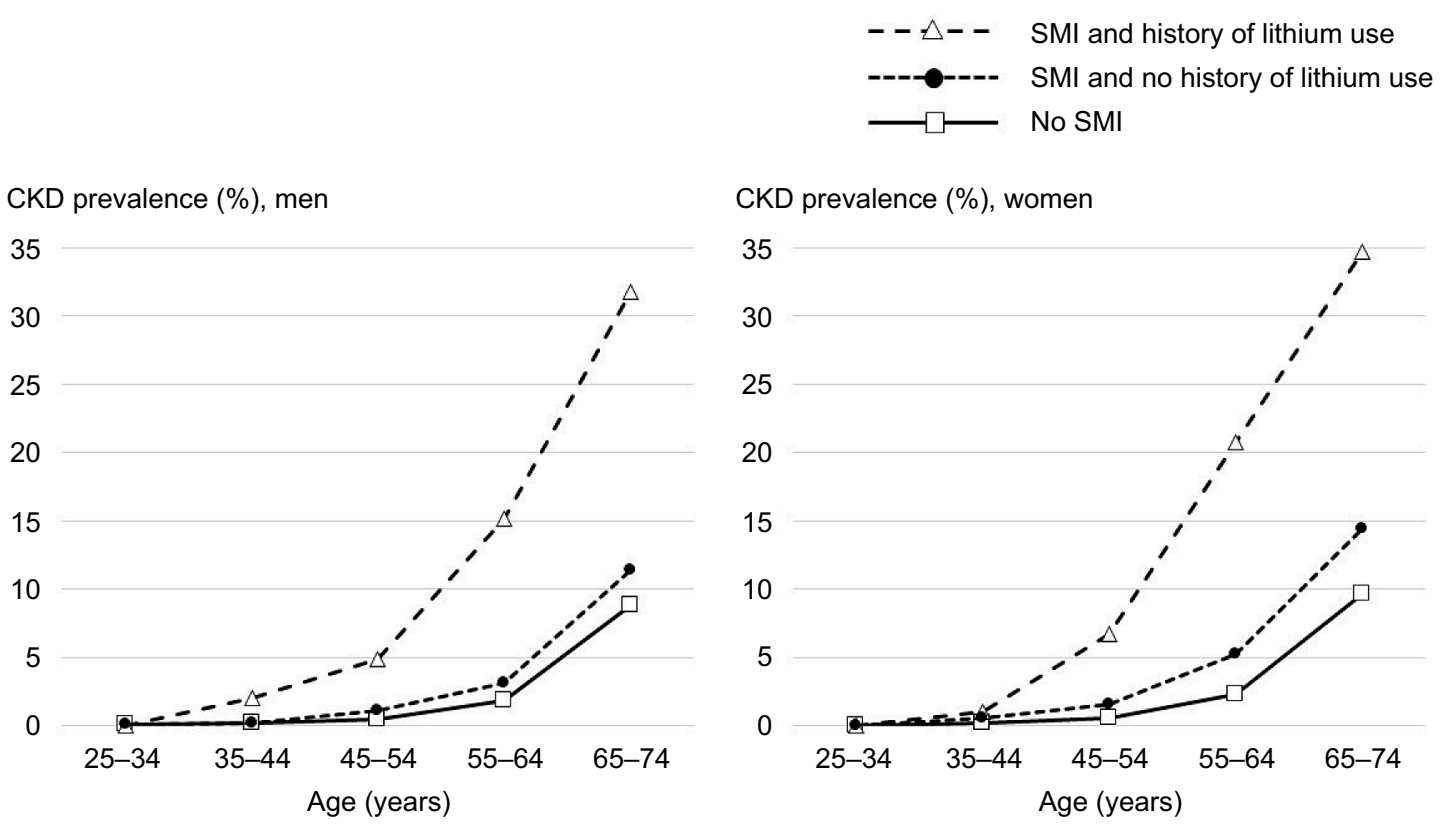

Figure 2 Prevalence of CKD in patients with and without SMI by age in men and women. Abbreviations: CKD, chronic kidney disease; SMI, severe mental illness.

Table 2 Adjusted logistic regression analyses of the association between SMI and chronic kidney disease

\begin{tabular}{llll}
\hline Model & \multicolumn{3}{l}{ Adjusted odds ratio (95\% Cl) for chronic kidney disease } \\
\cline { 2 - 4 } & Patients without SMI & $\begin{array}{l}\text { Patients with SMI and no } \\
\text { history of lithium use }\end{array}$ & $\begin{array}{l}\text { Patients with SMI and } \\
\text { history of lithium use }\end{array}$ \\
\hline Model I: adjusted by age and sex & & I.69 (I.56-I.83) & $7.13(6.47-7.85)$ \\
Model 2: Model I + adjusted by ethnicity, country, and & I (ref) & I.65 (I.52-I.79) & $7.13(6.48-7.86)$ \\
socioeconomic status & & & \\
Model 3: Model 2 + adjusted by smoking status & I (ref) & I.60 (I.48-I.74) & $6.91(6.27-7.61)$ \\
Model 4: Model 3 + adjusted by body mass index & I (ref) & I.53 (I.42-I.66) & $6.44(5.84-7.09)$ \\
Model 5: Model 4 + adjusted by diabetes & I (ref) & I.42 (I.3I-I.54) & $6.23(5.64-6.88)$ \\
Model 6: Model 5 + adjusted by other chronic diseases* & I (ref) & I.45 (I.34-I.58) & $6.49(5.84-7.21)$ \\
\hline
\end{tabular}

Note: *Hypertension, cardiovascular disease (myocardial infarction, chronic heart failure, peripheral arterial disease, and stroke), urological disease (vesicoureteral reflux, renal tract stone, and prostatic hypertrophy), systematic lupus erythematosus, and polycystic kidney disease.

Abbreviations: $\mathrm{Cl}$, confidence interval; ref, reference; SMI, severe mental illness.

Table 3 Recognition and management of CKD in CKD patients with and without SMI

\section{Indicator of CKD recognition/management}

Patients with biochemically defined CKD (not on RRT)

With SMI N = I,388,

n (\%)

1) Recorded diagnosis of CKD

2) Most recent blood pressure $\leq 140 / 90 \mathrm{mmHg}$

3) Prescription of renin-angiotensin system antagonists (among patients with hypertension and proteinuria diagnoses), numerator/denominator (\%)

4) Proteinuria/albuminuria testing in the past year

5) Prescription of statins
$976(70.3)$

I, II $12(80.1)$

$52 / 66(78.8)$

$421(30.3)$

$70 \mathrm{I}(50.5)$
Without SMI N = 47,225, n (\%)

$30,922(65.5)$

$35,705(75.6)$

$2,905 / 3,428(84.7)$

$15,263(32.3)$

$27,853(59.0)$

Abbreviations: CKD, chronic kidney disease; RRT, renal replacement therapy; SMI, severe mental illness.

without SMI is unlikely to be a substantial contributor to our results. Third, RRT is a rare outcome: the incidence of RRT in the UK is $\sim 100$ per million population. ${ }^{39}$ Combined with the cross-sectional design, this meant that, despite the large sample size, the number of patients with SMI and RRT was limited $(n=46)$. Although there was a statistically significant difference in the prevalence of RRT between patients with and without SMI, more detailed comparison (eg, stratification 
by age and sex) or analysis of risk factors for RRT was not possible with these numbers. Finally, our adjusted analyses suggested that differences in the prevalence of risk factors between patients with and without SMI did not completely explain the association between SMI and CKD. This finding may be due to additional unconsidered risk factors, differences in patient management, or residual confounding. For example, antipsychotics used for SMI have been suggested to cause acute kidney injury, ${ }^{40,41}$ which is a known risk factor for subsequent CKD. ${ }^{21}$ Furthermore, insufficient control - or later initiation - of treatment for the CKD risk factors (eg, high blood pressure, diabetes, and obesity) may lead to higher incidence of CKD in the SMI population; however, this information could not be adequately captured in this cross-sectional study. Using recorded CPRD diagnoses, moreover, means that the misclassification or underidentification of disease status (eg, diabetes and heart failure) is possible. We did not have any information on the use of lithium before the CPRD registration and, therefore, some patients may have been wrongly classified as not having used lithium. This also meant that we were unable to examine the association between the length or cumulative dose of lithium prescription and CKD.

\section{Comparison with other studies}

Few studies have examined the prevalence of CKD in the population with SMI. A small cross-sectional study in London, $\mathrm{UK}$, found a similar relative difference in CKD prevalence between those with and without SMI despite a less accurate CKD definition than in the present study. ${ }^{13}$ A Taiwanese cohort study showed that people with schizophrenia are more likely to develop CKD, ${ }^{25}$ whereas a cross-sectional analysis of Scottish primary care demonstrated that people with bipolar disorder had a higher prevalence of CKD than people without the condition. ${ }^{18}$ Several studies have focused on the prevalence or incidence of CKD in patients using lithium. A Swedish cohort study showed that the prevalence of CKD (defined as serum creatinine level $>150 \mu \mathrm{mol} / \mathrm{L}$ ) and ESRD requiring RRT was higher in people ever exposed to lithium than in the general population, ${ }^{42}$ whereas a Danish cohort study also showed that lithium prescription was associated with an increased rate of CKD diagnosis. ${ }^{26}$

The relative risk of lithium use for the prevalence of CKD in our study (the fully adjusted OR of 6.5) was larger than those estimated in previous studies: the adjusted hazard ratio by lithium for CKD was nearly 2 in a cohort study in Oxford, UK, ${ }^{43}$ and around 3 in the Danish cohort study. ${ }^{26}$ These differences can be explained by the different nature of the study population in each study. We compared people with lithium prescription for SMI and those without SMI in the general population, whereas the UK cohort study compared those with and without lithium use among people with at least two blood samplings in hospitals in Oxford, ${ }^{43}$ and the Danish study estimated the risk of lithium prescription in the population with a single manic episode or bipolar disorder. ${ }^{26}$ Therefore, our comparison group is healthier than in the other studies, although again, ascertainment bias may play a role to some extent in our study.

The management of CKD includes prevention of cardiovascular events. Several previous studies have focused on inequalities in medication use for cardiovascular risk between patients with and without SMI. Studies from the UK showed that statin prescribing is lower in patients with schizophrenia and bipolar disorder than in the general population, although their cardiovascular risks are higher. ${ }^{16,18}$ A US study suggests that prescribing of renin-angiotensin system antagonists and statins is suboptimal in patients with SMI and type 2 diabetes. ${ }^{17}$ Our finding of lower prescribing of statins among patients with SMI and CKD, compared to the general population, are consistent with these earlier studies.

\section{Explanation of findings and clinical relevance}

CKD is strongly and independently associated with mortality and cardiovascular risk. ${ }^{20}$ Therefore, the higher prevalence of $\mathrm{CKD}$ we have established may contribute to the known shorter life expectancy in people with SMI. Moreover, we have shown that the difference in CKD prevalence between patients with and without SMI increased with age. It is possible that progressive accumulation and biological effect of CKD risk factors in patients with SMI (eg, obesity, smoking, and diabetes) leads to an increased incidence of CKD at an older age.

We have confirmed a higher burden of CKD risk factors among patients with SMI, and adjustment for these partially explained the association between SMI and CKD. However, many questions about the cause of the higher prevalence of CKD remain unanswered. Our snapshot of GP's management of patients with SMI suggests that CKD is more commonly coded as a diagnosis than in the general population. The extent to which this is driven by incentivized management schemes and regular testing of renal function for patients with SMI - particularly those prescribed lithium and other psychotropic medications - is unknown. Whereas blood pressure appeared better controlled among SMI patients, this may reflect different underlying renal pathologies associated with 
lower rates of hypertension (eg, interstitial nephritis related to lithium). Other aspects of management - proteinuria testing as well as prescription of statins and renin-angiotensin system antagonists - were lower among patients with SMI.

Of greatest concern is our finding of the substantial increase in the prevalence of RRT among patients with SMI. The development of ESRD results in markedly reduced quality of life and psychological stress. Renal transplantation is associated with better quality of life and, possibly, longer survival. ${ }^{44}$ However, our results showed that the proportion of patients receiving kidney transplantation was substantially lower in patients with SMI than those without. It is important to understand why this difference arises and ensure that there are no inappropriate barriers to the consideration for renal transplantation for people with SMI.

\section{Conclusion}

We found that the prevalence of CKD and RRT was substantially higher in patients with SMI as compared to the general population. There was a greater burden of risk factors among patients with SMI, but these did not fully explain the increased prevalence of CKD. Further information about the management of CKD in SMI patients such as referral to specialist care and management of comorbidities is needed to identify opportunities for prevention of CKD and its progression.

\section{Acknowledgments}

Masao Iwagami is supported by the Honjo International Scholarship Foundation. Laurie A Tomlinson is funded by a Wellcome Intermediate Clinical Fellowship (WT101143MA). Liam Smeeth is supported by a Wellcome Senior Research Fellowship in Clinical Science (grant number 098504/Z/12/Z). David PJ Osborn is supported by the UCLH NIHR Biomedical Research Centre and he was also in part supported by the National Institute for Health Research (NIHR) Collaboration for Leadership in Applied Health Research and Care (CLAHRC) North Thames at Bart's Health NHS Trust. The funders had no role in the execution of this study or in the interpretation of results.

\section{Disclosure}

The authors report no conflicts of interest in this work.

\section{References}

1. Chang CK, Hayes RD, Perera G, et al. Life expectancy at birth for people with serious mental illness and other major disorders from a secondary mental health care case register in London. PLoS One. 2011;6(5): e19590.
2. Dembling BP, Chen DT, Vachon L. Life expectancy and causes of death in a population treated for serious mental illness. Psychiatr Serv. 1999;50(8):1036-1042.

3. Hannerz $\mathrm{H}$, Borgå $\mathrm{P}$, Borritz $\mathrm{M}$. Life expectancies for individuals with psychiatric diagnoses. Public Health. 2001;115(5):328-337.

4. Roshanaei-Moghaddam B, Katon W. Premature mortality from general medical illnesses among persons with bipolar disorder: a review. Psychiatr Serv. 2009;60(2):147-156.

5. Crump C, Winkleby MA, Sundquist K, Sundquist J. Comorbidities and mortality in persons with schizophrenia: a Swedish national cohort study. Am J Psychiatry. 2013;170(3):324-333.

6. Brown S, Kim M, Mitchell C, Inskip H. Twenty-five year mortality of a community cohort with schizophrenia. Br J Psychiatry. 2010;196(2): 116-121.

7. Osborn DP, Levy G, Nazareth I, Petersen I, Islam A, King MB. Relative risk of cardiovascular and cancer mortality in people with severe mental illness from the United Kingdom's General Practice Rsearch Database. Arch Gen Psychiatry. 2007;64(2):242-249.

8. Osby U, Correia N, Brandt L, Ekbom A, Sparén P. Mortality and causes of death in schizophrenia in Stockholm county, Sweden. Schizophr Res. 2000;45(1-2):21-28.

9. Osby U, Brandt L, Correia N, Ekbom A, Sparén P. Excess mortality in bipolar and unipolar disorder in Sweden. Arch Gen Psychiatry. 2001;58(9):844-850.

10. Osborn DP, Nazareth I, King MB. Risk for coronary heart disease in people with severe mental illness: cross-sectional comparative study in primary care. Br J Psychiatry. 2006;188:271-277.

11. Daumit GL, Clark JM, Steinwachs DM, Graham CM, Lehman A, Ford DE. Prevalence and correlates of obesity in a community sample of individuals with severe and persistent mental illness. J Nerv Ment Dis. 2003;191(12):799-805.

12. McEvoy JP, Meyer JM, Goff DC, et al. Prevalence of the metabolic syndrome in patients with schizophrenia: baseline results from the Clinical Antipsychotic Trials of Intervention Effectiveness (CATIE) schizophrenia trial and comparison with national estimates from NHANES III. Schizophr Res. 2005;80(1):19-32.

13. Woodhead C, Ashworth M, Schofield P, Henderson M. Patterns of physical co-/multi-morbidity among patients with serious mental illness: a London borough-based cross-sectional study. BMC Fam Pract. 2014;15:117.

14. Osborn DP, Baio G, Walters K, et al. Inequalities in the provision of cardiovascular screening to people with severe mental illnesses in primary care: cohort study in the United Kingdom THIN Primary Care Database 2000-2007. Schizophr Res. 2011;129(2-3):104-110.

15. Smith DJ, Langan J, McLean G, Guthrie B, Mercer SW. Schizophrenia is associated with excess multiple physical-health comorbidities but low levels of recorded cardiovascular disease in primary care: cross-sectional study. BMJ Open. 2013;3(4):e002808.

16. Hippisley-Cox J, Parker C, Coupland C, Vinogradova Y. Inequalities in the primary care of patients with coronary heart disease and serious mental health problems: a cross-sectional study. Heart. 2007;93(10):1256-1262.

17. Kreyenbuhl J, Medoff DR, Seliger SL, Dixon LB. Use of medications to reduce cardiovascular risk among individuals with psychotic disorders and Type 2 diabetes. Schizophr Res. 2008;101(1-3):256-265.

18. Smith DJ, Martin D, McLean G, Langan J, Guthrie B, Mercer SW. Multimorbidity in bipolar disorder and undertreatment of cardiovascular disease: a cross sectional study. BMC Med. 2013;11:263.

19. National Kidney Foundation. K/DOQI clinical practice guidelines for chronic kidney disease: evaluation, classification, and stratification. $\mathrm{Am}$ J Kidney Dis. 2002;39(2 Suppl 1):S1-S266.

20. Go AS, Chertow GM, Fan D, McCulloch CE, Hsu CY. Chronic kidney disease and the risks of death, cardiovascular events, and hospitalization. N Engl J Med. 2004;351(13):1296-1305.

21. National Institute for Health and Care Excellence. Chronic kidney disease in adults: assessment and management. Available from: https:// www.nice.org.uk/guidance/cg182. Accessed October 22, 2017. 
22. Dixon L, Weiden P, Delahanty J, et al. Prevalence and correlates of diabetes in national schizophrenia samples. Schizophr Bull. 2000;26(4): 903-912.

23. Markowitz GS, Radhakrishnan J, Kambham N, Valeri AM, Hines WH, D'Agati VD. Lithium nephrotoxicity: a progressive combined glomerular and tubulointerstitial nephropathy. J Am Soc Nephrol. 2000;11(8): 1439-1448.

24. Hayes JF, Marston L, Walters K, Geddes JR, King M, Osborn DP. Adverse renal, endocrine, hepatic, and metabolic events during maintenance mood stabilizer treatment for bipolar disorder: a population-based cohort study. PLoS Med. 2016;13(8):e1002058.

25. Tzeng NS, Hsu YH, Ho SY, et al. Is schizophrenia associated with an increased risk of chronic kidney disease? A nationwide matched-cohort study. BMJ Open. 2015;5(1):e006777.

26. Kessing LV, Gerds TA, Feldt-Rasmussen B, Andersen PK, Licht RW. Use of lithium and anticonvulsants and the rate of chronic kidney disease: a nationwide population-based study. JAMA Psychiatry. 2015;72(12):1182-1191.

27. Health and Social Care Information Centre. Quality and outcomes framework. Available from: www.Hscic.Gov.Uk/qof. Accessed October $22,2017$.

28. Herrett E, Gallagher AM, Bhaskaran K, et al. Data Resource Profile: Clinical Practice Research Datalink (CPRD). Int J Epidemiol. 2015;44(3): 827-836.

29. Nazareth I, King M, Haines A, Rangel L, Myers S. Accuracy of diagnosis of psychosis on general practice computer system. BMJ. 1993;307(6895):32-34.

30. Levey AS, Stevens LA, Schmid CH, et al; CKD-EPI (Chronic Kidney Disease Epidemiology Collaboration). A new equation to estimate glomerular filtration rate. Ann Intern Med. 2009;150(9):604-612.

31. Iwagami M, Tomlinson LA, Mansfield KE, et al. Validity of estimated prevalence of decreased kidney function and renal replacement therapy from primary care electronic health records compared with national survey and registry data in the United Kingdom. Nephrol Dial Transplant. 2017;32(Suppl 2):ii142-ii150.

32. Hippisley-Cox J, Coupland C. Derivation and validation of updated QFracture algorithm to predict risk of osteoporotic fracture in primary care in the United Kingdom: prospective open cohort study. $B M J$. 2012;344:e3427.
33. Hippisley-Cox J, Coupland C. Predicting risk of upper gastrointestinal bleed and intracranial bleed with anticoagulants: cohort study to derive and validate the QBleed scores. BMJ. 2014;349:g4606.

34. Department for Communities and Local Government. English indices of deprivation. Available from: www.Gov.Uk/government/collections/ english-indices-of-deprivation. Accessed October 22, 2017.

35. NHS Staffordshire Commissioning Support Unit. Recommended read codes for quality \& outcomes framework. Available from: https://www. pcc-cic.org.uk/article/qof-read-codes-v28. Accessed October 22, 2017.

36. National Institute for Health and Care Excellence. Cardiovascular disease: risk assessment and reduction, including lipid modification. Available from: https://www.nice.org.uk/guidance/cg181. Accessed October 22, 2017.

37. Ochoa S, Usall J, Cobo J, Labad X, Kulkarni J. Gender differences in schizophrenia and first-episode psychosis: a comprehensive literature review. Schizophr Res Treatment. 2012;2012:916198.

38. Quality and Outcomes Framework guidance for GMS contract 2013/14. Available from: https:/www.bma.org.uk/-/media/files/pdfs/practical\%20advice\%20at\%20work/contracts/gpqofguidance20132014.pdf. Accessed October 22, 2017.

39. Rao A, Casula A, Castledine C. UK Renal Registry 17th Annual Report: Chapter 2 UK Renal Replacement Therapy Prevalence in 2013: Nationa and Centre-specific Analyses. Nephron. 2015;129(Suppl 1):31-56.

40. Jiang Y, McCombs JS, Park SH. A retrospective cohort study of acute kidney injury risk associated with antipsychotics. CNS Drugs. 2017;31(4):319-326.

41. Hwang YJ, Dixon SN, Reiss JP, et al. Atypical antipsychotic drugs and the risk for acute kidney injury and other adverse outcomes in older adults: a population-based cohort study. Ann Intern Med. 2014;161(4): 242-248.

42. Bendz H, Schön S, Attman PO, Aurell M. Renal failure occurs in chronic lithium treatment but is uncommon. Kidney Int. 2010;77(3): 219-224.

43. Shine B, McKnight RF, Leaver L, Geddes JR. Long-term effects of lithium on renal, thyroid, and parathyroid function: a retrospective analysis of laboratory data. Lancet. 2015;386(9992):461-468.

44. Thiruchelvam PT, Willicombe M, Hakim N, Taube D, Papalois V. Renal transplantation. BMJ. 2011;343:d7300.
Clinical Epidemiology

\section{Publish your work in this journal}

Clinical Epidemiology is an international, peer-reviewed, open access, online journal focusing on disease and drug epidemiology, identification of risk factors and screening procedures to develop optimal preventative initiatives and programs. Specific topics include: diagnosis, prognosis, treatment, screening, prevention, risk factor modification,

Submit your manuscript here: https://www.dovepress.com/clinical-epidemiology-journal
Dovepress

systematic reviews, risk and safety of medical interventions, epidemiology and biostatistical methods, and evaluation of guidelines, translational medicine, health policies and economic evaluations. The manuscript management system is completely online and includes a very quick and fair peer-review system, which is all easy to use. 